\title{
A case of advanced systemic sclerosis with severe GERD successfully treated with acotiamide
}

Ryo Kato ${ }^{1}$, Kiyokazu Nakajima ${ }^{1,2^{*}}$, Tsuyoshi Takahashi ${ }^{1}$, Yasuhiro Miyazaki ${ }^{1}$, Tomoki Makino ${ }^{1}$, Yukinori Kurokawa', Makoto Yamasaki ${ }^{1}$, Shuji Takiguchi ${ }^{1}$, Masaki Mori $^{1}$ and Yuichiro Doki ${ }^{1}$

\begin{abstract}
The majority of systemic sclerosis (SSc) patients have gastrointestinal tract involvement, but therapies of prokinetic agents are usually unsatisfactory. Patients are often compromised by the use of steroid; therefore, a surgical indication including fundoplication has been controversial. There is no report that advanced SSc with severe gastroesophageal reflux disease (GERD) is successfully treated with acotiamide, which is the acetylcholinesterase (AChE) inhibitor designed for functional dyspepsia (FD). We report a 44-year-old woman of SSc with severe GERD successfully treated with acotiamide. She had received medical treatment in our hospital since 2003. She had been aware of the significant gastroesophageal reflux symptoms (e.g., heartburn, chest pain, and dysphagia) due to the development of esophageal hardening associated with SSc since 2014. As a result of upper gastrointestinal series, upper gastrointestinal endoscopy, and 24-h pH monitoring and frequency scale for the symptoms of the GERD (FSSG) scoring, she has been diagnosed with GERD associated with SSc. First of all, she started to take prokinetic agents Rikkunshito and mosapride and proton pump inhibitor; there was no change in reflux symptoms. So, we started to prescribe her the acotiamide.

After oral administration started, reflux symptoms have been improved. Five months after oral administration, FSSG score, a questionnaire for evaluation of the symptoms of GERD, was improved. Since its introduction of acotiamide, the patient has kept free from symptoms for 6 months.
\end{abstract}

Keywords: acotiamide, systemic sclerosis, gastroesophageal reflux disease (GERD), functional dyspepsia (FD), frequency scale for the symptoms of the GERD (FSSG)

\section{Background}

Systemic sclerosis (SSc) is a multisystem and chronic disease characterized by abnormalities of small blood vessels and fibrosis of the skin and internal organs. SSc, when advanced, is often compromised with severe gastroesophageal reflux disease (GERD), which may be lethal in a worst-case scenario. A wide variety of medication has been used [1-5]; however, none of them are promising for patients with SSc. In addition, patients with

\footnotetext{
* Correspondence: knakajima@gesurg.med.osaka-u.ac.jp

Ryo Kato is the first author.

'Department of Gastroenterological Surgery, Graduate School of Medicine, Osaka University, 2-2, E-2, Yamadaoka, Suita, Osaka 565-0871, Japan

${ }^{2}$ Division of Next Generation Endoscopic Intervention (Project ENGINE), Global Center for Medical Engineering and Informatics, Center of Medical Innovation and Translational Research, Osaka University, 2-2, Yamadaoka, Suita, Osaka 565-0871, Japan
}

\section{Case presentation}

A 44-year-old woman of SSc had received medical treatment in our hospital since 2003. She had been aware of the significant gastroesophageal reflux symptoms and esophagus stasis due to the development of esophageal hardening associated with SSc since 2014. On physical examination, cachexia, a "mouse face" appearance and ulceration in the distal phalanges were identified. The 
abnormal build-up of fibrous tissue in the skin can cause the skin to tighten so severely that her fingers curl and lose their mobility (Fig. 1). Because she had been aware of the worsening of gastroesophageal reflux symptoms, she received a medical examination from this department. Upper gastrointestinal series revealed no expansion and meandering esophagus, and reflux into the esophagus in the Trendelenburg position (Fig. 2). The upper gastrointestinal endoscopy showed reflux esophagitis of Los Angeles classification grade $\mathrm{C}$ and esophagus residue (Fig. 3).

A 24-h esophageal $\mathrm{pH}$ monitoring revealed significant acid reflux: the number of refluxes was 81 times, $\mathrm{pH}$ was below 4.0 for $32.1 \%$, mean $\mathrm{pH}$ was 4.55 , and DeMeester score (normal <14.75) was 117.5 (Fig. 4). Symptoms of gastroesophageal reflux disease (frequency scale for the symptoms of the GERD (FSSG)) score, a questionnaire evaluating the symptoms of GERD, was 34 points [6] (maximum 48 points). As a result of these tests, she has been diagnosed with GERD associated with SSc. Treatment with Rikkunshito and mosapride, which are prokinetic agents, and proton pump inhibitor was started. However, her symptoms were not improved. Therefore, we started the acotiamide on June 2015, which was a new drug originally designed for FD. Since then, her symptoms which were heartburn, burp, and nausea after a meal were improved. Five months after acotiamide was started, the FSSG score was reduced to 21 points (Fig. 5). However, the results of 24-h esophageal $\mathrm{pH}$ monitoring showed worsening acid reflux: the number of refluxes was 152 times, $\mathrm{pH}$ was below 4.0 for $60.5 \%$, mean pH was 3.73, and DeMeester score (normal <14.75) was 211.6 (Fig. 4). The upper gastrointestinal series and upper gastrointestinal endoscopy did not change.

Since the introduction of acotiamide, the patient has been free from symptoms to date.



Fig. 1 The abnormal build-up of fibrous tissue in the skin can cause the skin to tighten so severely that fingers curl and lose their mobility in SSC

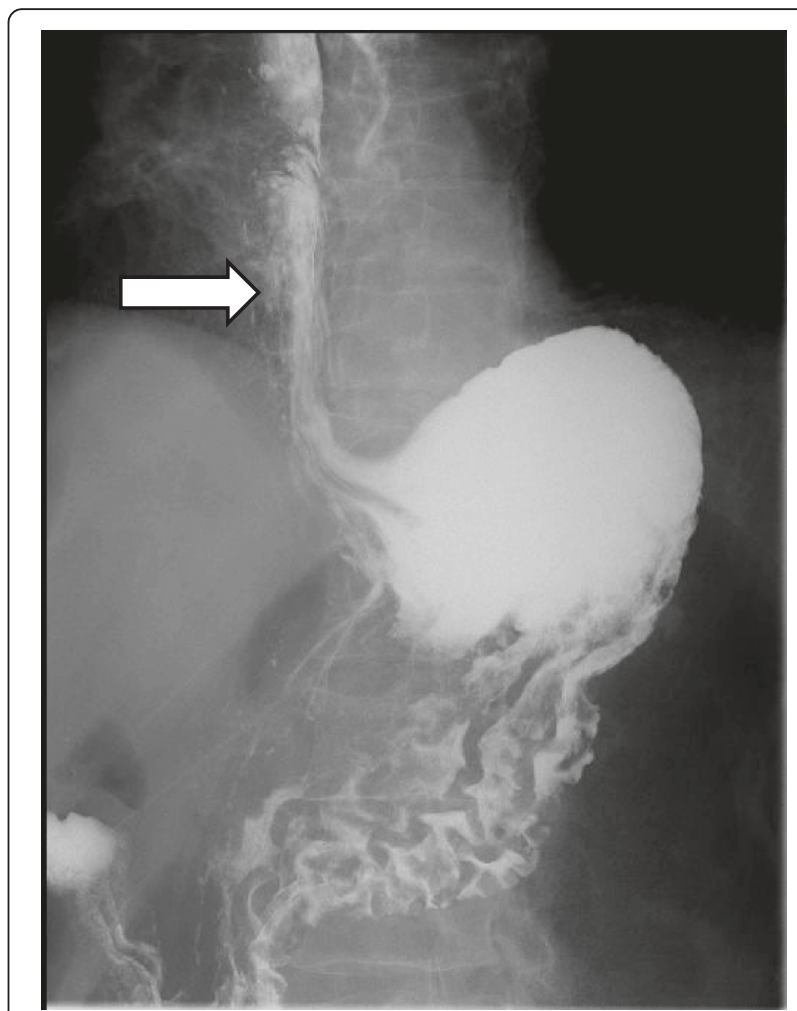

Fig. 2 Upper gastrointestinal series revealed no expansion and meandering esophagus and reflux into the esophagus in the Trendelenburg position

\section{Discussion}

In 1994, Sjogren proposed a progression of SSc with gastrointestinal involvement, vascular damage, neurogenic impairment, and myogenic dysfunction with the replacement of normal smooth muscle by collagens fibrosis and atrophy [7]. It is distinguished in diffuse cutaneous SSc (dcSSc) or limited cutaneous SSc (lcSSc) whether skin hardening exceeds an elbow or a knee [8]. Next to the skin, the gastrointestinal tract is the second most common site of SSc organ damage that can affect patients with lcSSc and dcSSc [9]. It affects the gastrointestinal tract in more than $80 \%$ of patients. Reflux esophagitis is found in $50-90 \%$ of SSc patients [7, 10].

The common characteristics of GERD seen in SSc patients are as follows. The upper GI series often shows peristaltic decrease and expansion of the lower esophagus. The upper gastrointestinal endoscopy shows linear redness and erosion of the lower esophagus caused by the reflux, which often merges with the Barett esophagus. In addition, the merger frequency of esophageal cancer is often in SSc. Esophageal dysmotility leads to impaired acid clearance, and 24-h monitoring shows prolongation of esophageal exposure time to gastric acid [11].

Its treatment, either medical or surgical, has been still challenging. The major medical treatment option includes 


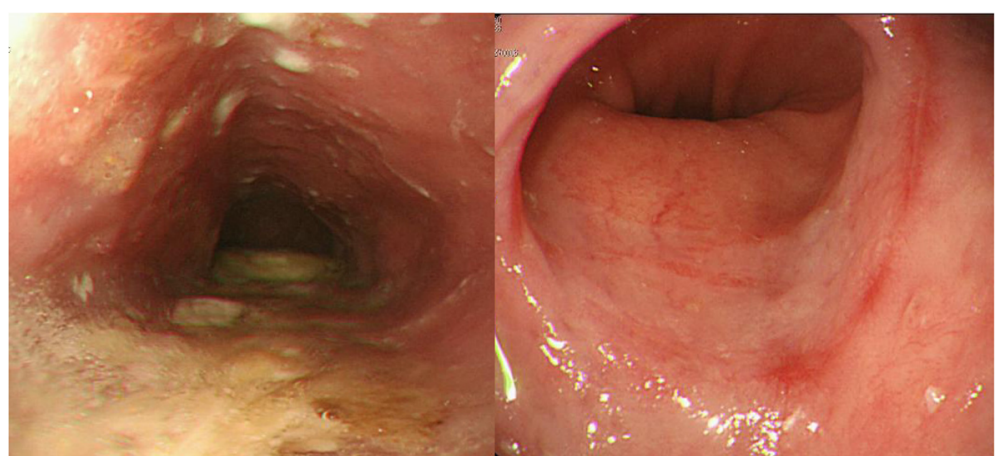

Fig. 3 The upper gastrointestinal endoscopy showed reflux esophagitis of Los Angeles classification grade C and esophagus residue

use of histamine-2 receptor antagonist (H2RA) and/or proton pomp inhibitor (PPI) for the purpose of controlling stimulation and inflammation of the esophageal mucosa by the gastric acid reflux [12-14]. Surgery, e.g., Nissen fundoplication, can be considered for drug-resistant reflux disease [15]. These medical/surgical treatments have been shown not as promising as those for reflux patients without SSc [16].

The last option for SSc patients with severe GERD is a group of prokinetic drugs. A wide variety of prokinetic agents have been used, such as mosapride citrate, metoclopramide, domperidone, erythromycin, octreotide, and dinoprost [1-5]. However, therapies of traditional prokinetic agents are usually unsatisfactory for severe GERD patients.

Acotiamide is the novel prokinetic agent basically designed for FD; it is the acetylcholinesterase (AChE) inhibitor. FD is a chronic disorder of sensation and movement (peristalsis) in the upper gastrointestinal tract. The acetylcholine (Ach) is released from cholinergic nerve terminals and lets the gastrointestinal tract shrink by binding to the muscarinic receptor of the gastrointestinal smooth muscle. It is thought that the Ach is broken down immediately by AChE and enterokinesis is regulated by this reaction. Acotiamide inhibits $\mathrm{AChE}$ and regulates the resolution of Ach. As a result, it increases the quantity of ACh available in the synaptic cleft and therefore improves enterokinesis [17].

We have prescribed a variety of traditional prokinetic agents, without obtaining even temporary relief of her symptoms. Therefore, we prescribed acotiamide. We performed upper gastrointestinal series, gastrointestinal endoscopy, 24-h $\mathrm{pH}$ monitoring, and the quality of life (QOL) scores for the assessment of gastroesophageal reflux before and after oral administration of acotiamide on this patient. No changes were observed before and after treatment in the upper GI series and upper gastrointestinal endoscopy. Acotiamide does not show the emission promoting effect on the normal gastric emptying in rats. However, prior research reports that acotiamide does

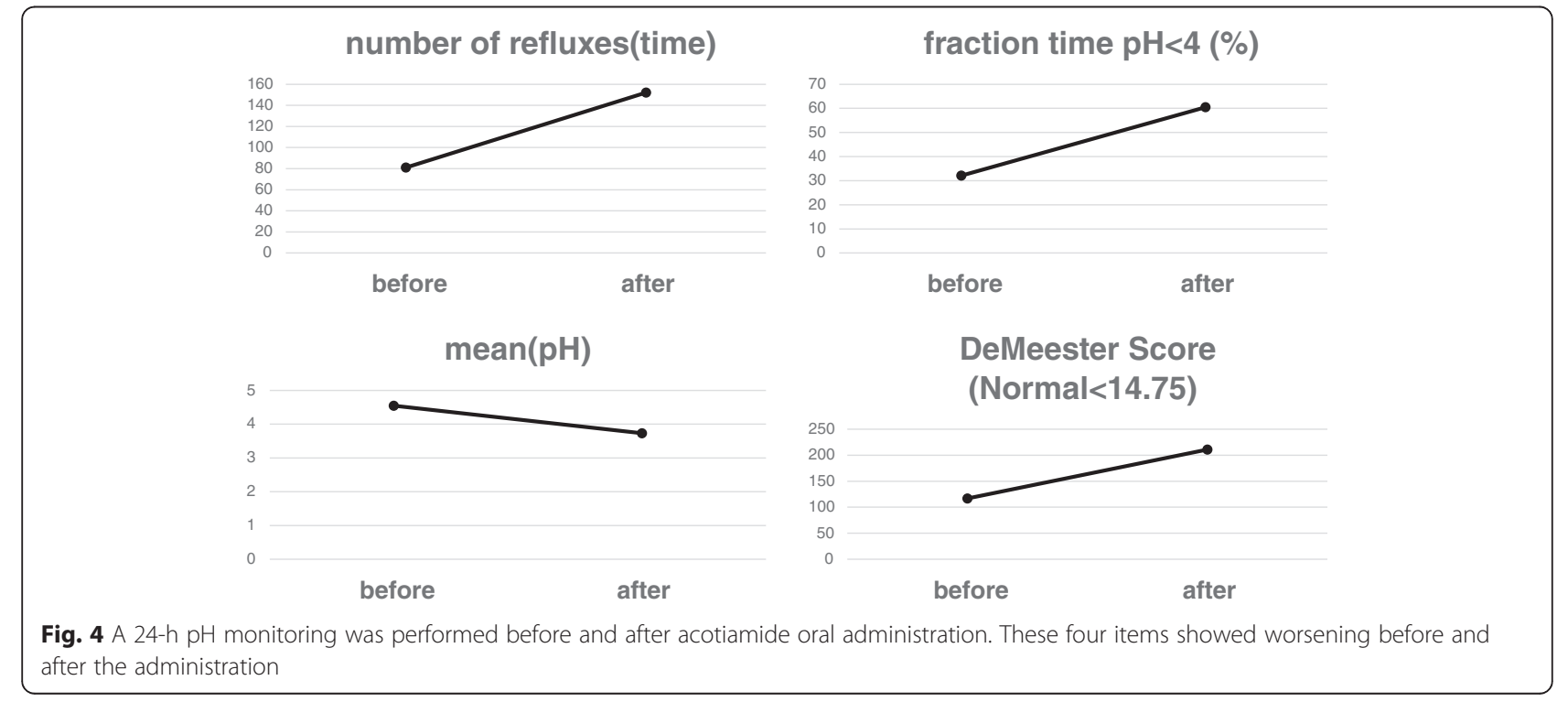




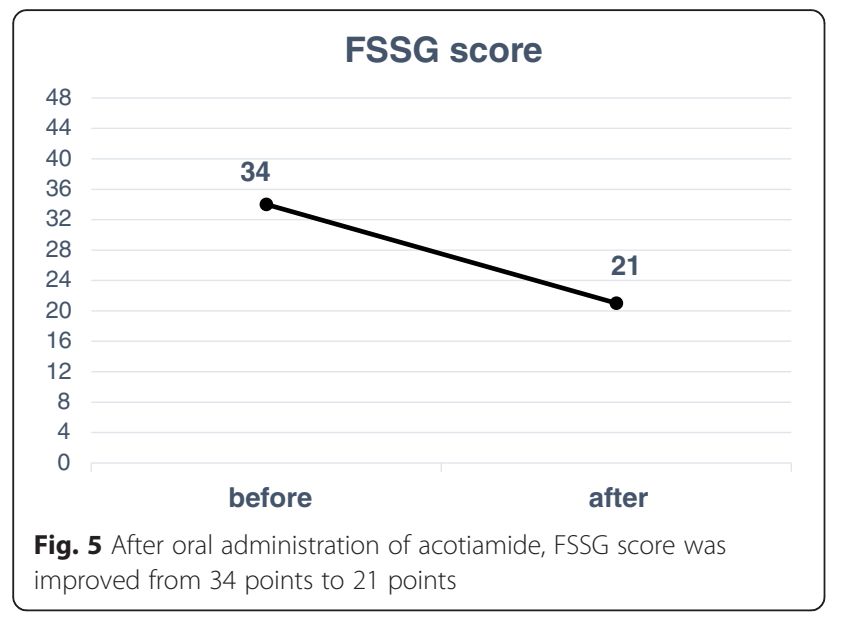

improve the gastric emptying during restraint stress. Acotiamide might have led to symptom improvement due to the suppressed response to stress by decreasing the expression of NmU, a stress-related gene in the hypothalamus, via the vagus nerve [18].We think this may be a partial reason why our patient showed improvement of her GI symptoms.

In the 24-h $\mathrm{pH}$ monitoring, DeMeester score even showed a worsening from 117 to 211. However, FSSG, which is one of the established QOL scoring system, showed improvement from total 34 to 21 points. FSSG is a questionnaire composed of 12 questions and classified into two groups, which are five items related to "dysmotility" symptoms and seven items to "acid reflux" symptoms. In our case, the score related to both symptoms were improved from 17 to 10 points and 17 points to 11 points, respectively. As we describe later, these improvements can be explained by the pharmacological effects of acotiamide on both the gastric and the esophageal functions.

In fact, this patient was clearly aware of the improvement of clinical symptoms, and QOL has been improved.

In our case, traditional prokinetic agents attempted prior to acotiamide were not effective. Only acotiamide showed substantial relief of the patient's symptom. We speculated this might be because of these two factors: (1) pharmacologically, acotiamide may not only affect the gastric emptying but also improve fundic accommodation of adaptive relaxation, and (2) acotiamide may directly act on the esophageal body and improve esophageal peristalsis. The authors have reached the above speculation based on the results of FSSG score: improvements of early satiety and chest discomfort. The question score "Do you feel full while eating meals?" was improved from 4 points to 2 points and "Do some things get stuck when you swallow?" was improved from 3 points to 2 points, respectively.
We just experienced one case; therefore, further accumulation of similar cases is definitely required. In our case, no objective improvement was observed on classical 24-h pH monitoring. The authors believe this examination might not be appropriate as an evaluation tool of GERD in patients with severe esophageal motor dysfunction like advanced SSc patients. Novais et al. reported that the 24-h abnormal $\mathrm{pH}$ tracings were classified into three types: (i) 24-h abnormal $\mathrm{pH}$, with a true GERD pattern, i.e., sharp sudden $\mathrm{pH}$ drops, reaching values below 3 and then returning to usual esophageal $\mathrm{pH}$ (pH 6-7) (Fig. 6a); (ii) 24-h abnormal pH with a pattern suggesting esophageal fermentation due to retained food, i.e., steady drop of $\mathrm{pH}$ not reaching values below 3.0 (Fig. 6b); and (iii) negative 24-h pH, i.e., presence of physiological reflux (reflux episodes occurring in less than $4.5 \%$ of total examining time) or zero reflux (absence of any episode of $\mathrm{pH}$ lower than 4.0) [19]. In a 24- $\mathrm{h} \mathrm{pH}$ monitoring after acotiamide was started, there were many frequent waveforms of (ii) than those of (i). The esophageal food fermentation may have affected the results of this case. The QOL score, including FSSG is likely to accurately reflect the symptoms of patients than 24-h pH monitoring. Future tasks are to perform a detailed study by using a new method of measuring such as high resolution manometry.

\section{Conclusions}

We have experienced a case of advanced SSc with severe GERD successfully treated with acotiamide. Acotiamide might become a help of advanced SSc with severe GERD patient whose surgical indication has been controversial.

\section{Consent}

Written informed consent was obtained from the patient for publication of this case report and accompanying

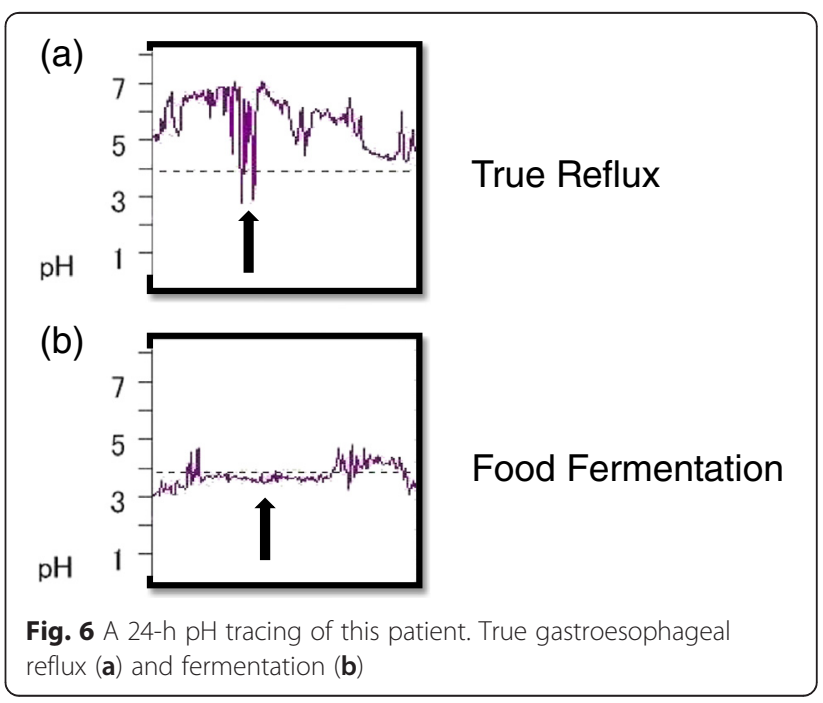


images. A copy of the written consent is available for review by the Editor-in-Chief of this journal.

\section{Abbreviations}

ACh: acetylcholine; AChE: acetylcholinesterase; FD: functional dyspepsia; FSSG: frequency scale for the symptoms of the GERD;

GERD: gastroesophageal reflux disease; SSc: systemic sclerosis.

\section{Competing interests}

The authors declare that they have no competing interests.

\section{Authors' contributions}

All authors participated in the management of the patient in this case report. KN is a chief surgeon of our hospital and supervised the case and also supervised the writing of the manuscript. YD is a chairperson of our department and supervised the entire process. All authors read and approved the final manuscript.

Received: 14 January 2016 Accepted: 6 April 2016

Published online: 13 April 2016

\section{References}

1. Johnson DA, Drane WE, Curran J, Benjamin SB, Chobanian SJ, et al. Metoclopramide response in patients with progressive systemic sclerosis. Effect on esophageal and gastric motility abnormalities. Arch Intern Med. 1987:147:1597-1601.1.

2. Smout AJ, Bogaard JW, Grade AC, ten Thije OJ, Akkermans LM, et al. Effects of cisapride, a new gastrointestinal prokinetic substance, on interdigestive and postprandial motor activity of the distal oesophagus in man. Gut. 1985;26:246-51.

3. Soudah $\mathrm{HC}$, Hasler WL, Owyang $\mathrm{C}$, et al. Effect of octreotide on intestinal motility and bacterial overgrowth in scleroderma. N Engl J Med. 1991:325:1461-7.

4. Dull JS, Raufman JP, Zakai MD, Strashun A, Straus EW, et al. Successful treatment of gastroparesis with erythromycin in a patient with progressive systemic sclerosis. Am J Med. 1990;89:528-30.

5. Tomomasa T, Kuroume T, Arai H, Wakabayashi K, Itoh Z, et al. Erythromycin induces migrating motor complex in human gastrointestinal tract. Dig Dis Sci. 1986;31:157-61.

6. Kusano M, Shimoyama Y, Sugimoto S, et al. Development and evaluation of FSSG: frequency scale for the symptoms of GERD. J Gastroenterol. 2004;39:888-91.

7. Sjögren RW. Gastrointestinal motility disorders in scleroderma. Arthritis Rheum. 1994;37:1265-82

8. LeRoy EC, Black C, Fleischmajer R. Scleroderma (systemic sclerosis): classification, subsets and pathogenesis. J Rheumatol. 1988;15(2):202-5.

9. Clements PJ, Becvar R, Drosos AA, Ghattas L, Gabrielli A. Assessment of gastrointestinal involvement. Clin Exp Rheumatol. 2003;21:S15-8.

10. Young MA, Rose S, Reynolds JC. Gastrointestinal manifestations of scleroderma. Rheum Dis Clin North Am. 1996:22(4):797-823.

11. Tasleem A, Qazi M, Jaswinder S, et al. Assessment of esophageal involvement in systemic sclerosis and morphea (localized scleroderma) by clinical, endoscopic, manometric and pH metric features: a prospective comparative hospital based study. BMC Gastroenterol. 2015;15:24.

12. Petrokubi RJ, Jeffries $\mathrm{GH}$. Cimtidine versus an acid in scleroderma with reflux esophagitis: a randomized double-blind controlled study. Gastroenterology. 1979;77:691-5

13. Hendel L, Aggestrup S, Stentoft P. Long-term ranitidine in progressive systemic sclerosis (scleroderma) with gastroesophageal reflux. Scan J Gastroenterol. 1986;21:799-805.

14. Wigley FM, Sule SD. Novel therapy in the treatment of scleroderma. Expert Opin Investig Drugs. 2001;10:31-48

15. Cicala M, Emerenziani S, Guarino MP, Ribolsi M. Proton pump inhibitor resistance, the real challenge in gastro-esophageal reflux disease. World J Gastroenterol. 2013;19(39):6529-35.

16. Carlson DA, Hinchcliff M, Pandolfino JE. Advances in the evaluation and management of esophageal disease of systemic sclerosis. Curr Rheumatol Rep. 2015;17(1):475.

17. Kawachi M, Matsunaga Y, Tanaka T. Acotiamide hydrochloride (Z-338) enhances gastric motility and emptying by inhibiting acetylcholinesterase activity in rats. Eur J Pharmacol. 2011;666:218-25.
18. Seto K et al. Acotiamide, hydrocholoride (Z-338), a novel prokinetics agent, restores delayed gastric emptying and feeding inhibition induced by restraint stress in rats. Neurogastroenterol Motil. 2008;20(9):1051-9.

19. Novais PA, Lemme EMO. 24-h pH monitoring patterns and clinical response after achalasia treatment with pneumatic dilation or laparoscopic Heller myotomy. Aliment Pharmacol Ther. 2010;32:1257-65.

\section{Submit your manuscript to a SpringerOpen ${ }^{\circ}$ journal and benefit from:}

- Convenient online submission

- Rigorous peer review

- Immediate publication on acceptance

- Open access: articles freely available online

- High visibility within the field

- Retaining the copyright to your article

Submit your next manuscript at $>$ springeropen.com 